\author{
D.-A. Clevert \\ E. M. Jung \\ K.-P. Jungius \\ K. Ertan \\ R. Kubale
}

\section{Value of tissue harmonic imaging (THI) and contrast harmonic imaging (CHI) in detection and characterisation of breast tumours}

Received: 17 October 2005

Revised: 7 April 2006

Accepted: 25 April 2006

Published online: 6 July 2006

(C) Springer-Verlag 2006
K.-P. Jungius

Department of Radiology, Oberwallis, Brig, Switzerland

K. Ertan

Department of Gynaecology and Obstetrics,

University of Homburg/Saar, Homburg, Germany

\section{D.-A. Clevert $(\bowtie)$}

Department of Clinical Radiology, University of Munich -

Campus Grosshadern,

Marchioninistr. 15,

81377 Munich, Germany

e-mail: Dirk.Clevert@med.uni-

muenchen.de

Tel.: +49-89-70953620

Fax: $+49-89-70958832$

R. Kubale $(\bowtie)$

Institute of Diagnostic Radiology,

Ringstr. 60-64,

66953 Pirmasens, Germany

Tel.: +49-6331-51560

Fax: +49-6331-515640

E. M. Jung

Department of Diagnostic and

Interventional Radiology,

Passau Hospital,

Passau, Germany

\begin{abstract}
The purpose of this study was to investigate the extent to which tissue harmonic imaging (THI), speckle reduction imaging (SRI), spatial compounding (SC) and contrast can improve detection and differentiation of breast tumours. We examined 38 patients (14 benign, 24 malignant tumours) with different combinations of THI, SRI and SC. The effect on delineation, margin, tissue differentiation and posttumoral phenomena was evaluated with a three-point score. Additionally, 100 not palpable tumours (diameters: 4-15 $\mathrm{mm}$ ) were examined by contrast harmonic imaging $(\mathrm{CHI})$ with power Doppler. After bolus injection $(0.5 \mathrm{ml}$
\end{abstract}

Optison), vascularisation and enhancement were observed for $20 \mathrm{~min}$. The best combination for detection of margin, infiltration, echo pattern and posterior lesion boundary was the combination of SRI level 2 with SC low. THI was helpful for lesions OF more than $1 \mathrm{~cm}$ depth. In native Power Doppler, vessels were found in 54 of 100 lesions. Within 5 min after contrast medium (CM) injection, marginal and penetrating vessels increased in benign and malignant tumours and central vessels mostly in carcinomas $(p<0.05)$. A diffuse CM accumulation was observed up to $20 \mathrm{~min}$ after injection in malignant tumours only $(p<0.05)$. THI, SRI and SC improved delineation and tissue differentiation. Second-generation contrast agent allowed detection of tumour vascularisation with prolonged enhancement.

Keywords Breast ultrasound - Tissue harmonic imaging $\cdot$ Spatial compounding $\cdot$ Contrast harmonic imaging

\section{Introduction}

As a technique complementary to mammography, highresolution ultrasound (US) is well accepted for differentiating between cystic and noncystic putative tumour lesions [1-11]. New developments such as tissue harmonic imaging (THI) and contrast harmonic imaging (CHI) have enhanced its value, particularly for primary detecting tumours in dense inhomogeneous mammary gland tissue, identifying mammographically occult tumours and appraising abnormalities in tissue architecture [8, 12-22]. Lesions that are only a few millimetres in size become detectable, and tumours can be characterised more precisely. Ultimately, the exact localisation of small tumours is a prerequisite for ultrasound-guided needle biopsy, vacuum biopsy or preoperative wire placement $[15,23-25]$. Harmonic imaging allows better visualisation of tissue architecture and facilitates recognition of parenchymal 
changes with tumour infiltration, making it easier to wire mark small lesions with THI [19, 26, 27].

For tumour characterisation, it is also essential to appraise vascularisation in a manner comparable to that achieved by dynamic contrast-medium-enhanced magnetic resonance imaging (MRI) [4, 12, 16, 17, 28-31]. Even if computed evaluation programmes with pixel analysis facilitate appraisal of tumour blood flow, native vascular US with power Doppler (PD) has proved to be relatively unreliable $[4,9,13,17,18,23,24,29]$. Contrast medium enhancement already enables more distinct visualisation of intratumoral and peritumoral vessels. However, the brief diagnostic time window after bolus injection of US contrast medium $(\mathrm{CM})$ is often a limiting factor. The combination of CHI with PD opens up promising vistas for appraisal of vascularisation. Preliminary investigations indicate that tumour detection, characterisation and US-guided intervention can be improved by using a $\mathrm{CM}$ of the second generation with modified $\mathrm{CHI}$ technique $[18,25,32]$. The aim of our study was to evaluate their potential benefits and limitations for detecting and differentiating breast tumours.

\section{Materials and methods}

\section{Patients and US}

A total of 138 patients (age: $25-79$ years, median 54 years) were investigated in a multicentre trial with complementary mammary diagnostic procedures over a 36 -month period (November 2002 to November 2005). All women gave informed written consent for their results to be used for programme research and evaluation. On the basis of US findings, each lesion was evaluated based on size, shape (ellipsoid, round, irregular), margins (smooth, macrolobulated, microlobulated, speculated, angulated, ill-defined), posterior sonographic artefacts (shadowing, through transmission, no posterior artefacts), echogenicity (hyperechogenic, isoechoic, hypoechoic), presence of calcifications and ductal extension. Investigations were supplemented by clinical examination and mammography in two planes. Ultrasound lesions were examined in three planes using PD and digital imaging with the GE Logiq 9 (GE Medical Systems, Milwaukee, WI, USA) US units. All investigations were carried out with a high-resolution multifrequency linear matrix array transducer (Linear Array M10L, 5-10 MHz) with the modalities of THI, spatial compounding (crossbeam), speckle reduction imaging (SRI) and contrast-medium-enhanced PD with $\mathrm{CHI}$ and additional three-dimensional (3D) imaging. Imaging documentation of digital raw data in single-image and short sequences was effected using a Picture and Communication System (PACS).
Study population

The patient population was divided into two groups. In group 1, we focused on the advantage of THI, SC and an adaptive algorithm for SRI. In group 2, we focused on CHI with PD.

\section{Group 1}

Thirty-eight patients (37-62 years) with 14 benign and 24 malignant tumours were examined in fundamental mode and with harmonic imaging with a high-frequency matrix-array transducer with the modalities of THI, SC and an adaptive algorithm for SRI. Prospectively, fundamental and tissue harmonic mode were used alone and in combination with all available levels of SC (cross beam: low, medium, high, maximum) and five levels of SRI. In patients without tumour lesions, criteria were differentiation of normal fibroglandular tissue from premammary and retromammary fat, including the Cooper's ligaments and ducts. Criteria that were evaluated for differential diagnosis in patients with lesions were margin, infiltration of malignant tumours, echo pattern with tissue components, calcifications, posterior lesion boundary and posterior acoustic features. After optimising system settings, a three-level score was applied to enable comparison of the quality of scans with fundamental imaging $(0=$ equal, $-1=$ inferior, $+1=$ superior).

\section{Group 2}

One hundred patients (25-76 years, median 53 years) comprised 29 probably benign [Breast Imaging Reporting and Data System (BI-RADS III)] and 71 malignant (BIRADS IV-V) tumours confirmed by vacuum-assisted biopsy in 100/100 lesions and 78/100 cases additionally in the course of surgery. Tumour diameters varied between 4 and $15 \mathrm{~mm}$ (mean: $9 \mathrm{~mm}$ ). Histological confirmation was obtained in all cases. All patients were comparatively investigated with fundamental B scan, THI and CHI with PD. After the B scan and evaluation of vascularisation in native PD, we used an echo signal amplifier of the second generation with low mechanical index (MI) technology. A bolus injection of Optison was administered $(0.5 \mathrm{ml}$ Optison diluted with $20 \mathrm{ml}$ with $\mathrm{NaCl}$ solution and subsequent injection of another $20 \mathrm{ml} \mathrm{NaCl}$ ). The bolus of CM was injected intravenously, and spreading of the contrast enhancement and washout in the tumours were followed for at least $20 \mathrm{~min}$. A low MI (0.15-0.25) was chosen to avoid early destruction of the microbubbles.

$\mathrm{CM}$ enhancement was appraised for up to $20 \mathrm{~min}$. Moreover, additional 3D evaluations of the cine sequences were also possible retrospectively in consequence of the archiving of dynamic digital images of up to 180 single 
images and 10- loop. The images archived in B scan and THI could also be processed retrospectively, with various phases of SRI, which enabled marginal contours of the tumours to be highlighted by homogenising tissue structures. The patients were given detailed information before each injection of CM, particularly with respect to a possible predisposition to allergic reactions. Written consent was obtained. Studies were performed only in patients with normal renal function. Approval had been obtained from the hospital's ethics committee.

\section{Histopathological correlation}

Histopathological correlation was determined based on surgical and biopsy findings. Tumour size, grade, histological subtype and presence of invasion were documented. Pathological imaging correlation was performed in conjunction with both pathologist and breast imagers with regard to lesion location and size to ensure that the imaged lesions were evaluated histologically.

\section{Breast biopsy}

US-guided biopsies were performed as vacuum-assisted biopsies, mostly with an 11-gauge needle. Up to 12 representative tissue samples were taken. When there were malignant findings, US-guided wire marking was mostly undertaken before surgical excision. Afterwards, follow-up examinations were carried out at 6-month intervals.
Statistical analysis

We carried out statistical analyses:

1. Elementary statistics were computed.

2. Fisher's two-tailed exact test was applied for statistical assessment of powers for discriminating different vascularisation and comparison of benign tumours versus carcinomas after the use of Optison.

\section{Results}

Group1

THI has no effect on image quality in the near field. THI needs at least $1 \mathrm{~cm}$ of tissue penetration. The effect is best seen in the middle and far field, especially around the focus zone. Cutis and the first $10 \mathrm{~mm}$ of fat and Cooper's ligaments are already seen in fundamental mode, with better visibility and delineation with SC (cross beam: level medium) and SRI. The reflex of the anterior mammary fascia appears thinner and brighter with SC (cross beam: level medium to high) than in fundamental imaging. Ducts in deeper areas are best visualised with a combination of SC and THI. The main disadvantage is a significant reduction of the frame rate caused by SC, especially when THI is possible in addition. The first impression from image analysis of all combinations of SC and SRI was that higher levels of SC could be avoided by using, in addition, an SRI level of 1 or 2, giving the same advantage over the
Fig. 1 Effect on delineation of tumour margin and tissue differentiation. On the left side is fundamental mode without spatial averaging and tissue harmonic imaging (THI). On the right side $\mathrm{THI}+$ cross beam level low + speckle reduction imaging (SRI) 2. a Improvement of delineation of tumour margin, especially if the tumour border is perpendicular to the ultrasound beam (fibroadenoma). b Improvement of tissue differentiation (haematoma)
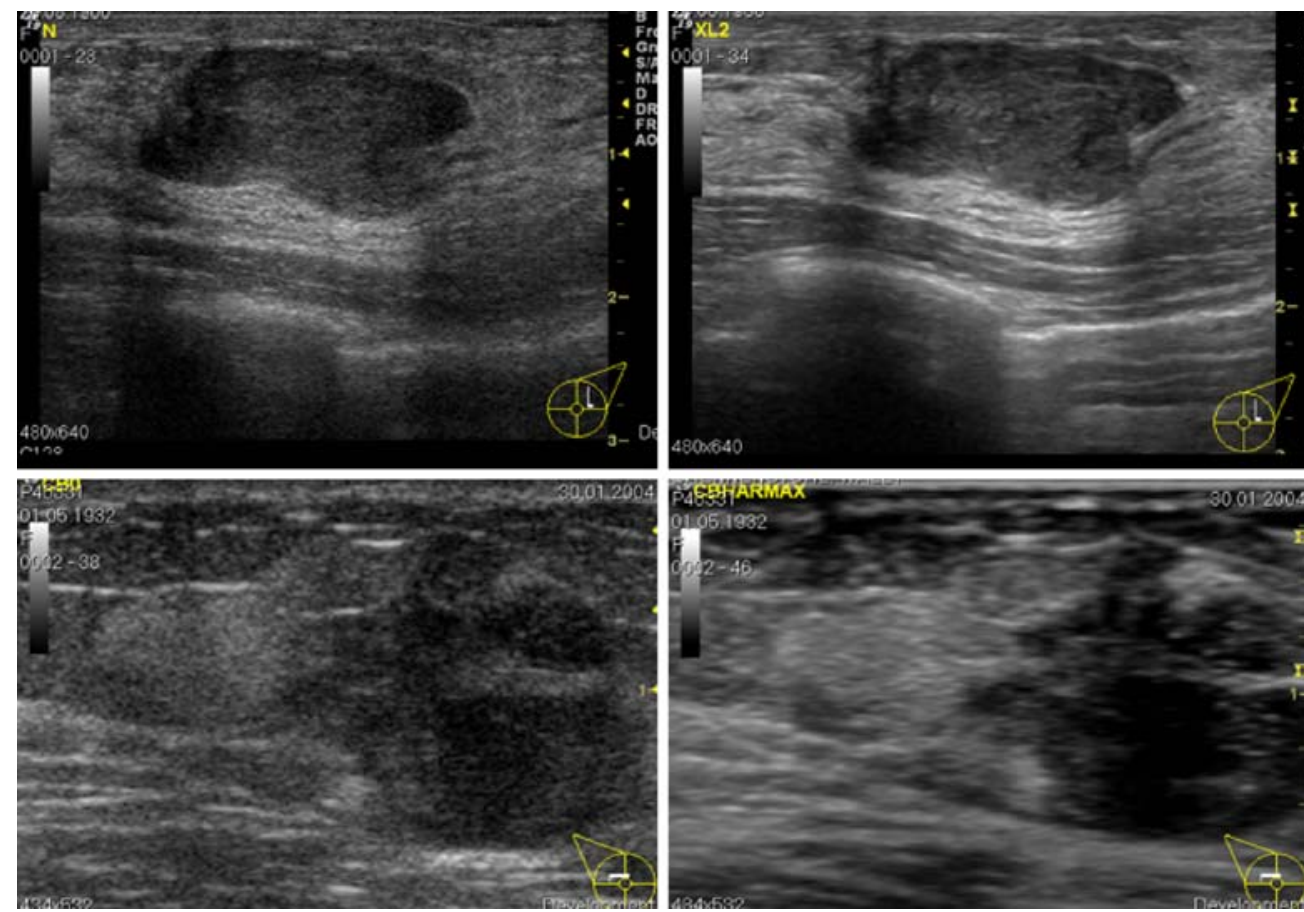
fundamental image with less reduction of frame rate. The best compromise for screening examinations of breast tissue is the combination of SC (level low) and SRI (level 1 or 2) with THI in small breasts. Fat appears slightly more echogenic, which potentially helps to define small tumours. The main limitation of THI was the reduced penetration (16\% of our patients). In patients with large breasts and abundant fibrous tissue, fundamental imaging should be preferred.

\section{Lesion detection and differentiation}

In four out of five cysts, THI clears artifactual echoes caused by reverberations, noise and speckle so that even small intracystic lesions could be depicted. Best visibility was achieved with a combination of THI, SC (level low) and SRI 2, giving clear cyst borders and the intracystic lesion. Using SC in two out of five cysts, the typical dorsal enhancement caused by sound transmission is less intense, as expected from the fundamental mode. Fibroadenomas were best detected with a combination of THI with SC (level medium) and SRI (2), showing better margin delineation, even in the edges. Better differentiation of tissue components and tumour infiltration could be achieved by the same combination (Fig. 1). Solid nodules generally appear more hypoechoic compared with sur- rounding breast tissue, which affords a better chance of detection [33].

Calcifications detected in mammography were seen in US in six out of ten patients. Visibility was increased, especially after using the combination of THI and SRI or SC and SRI (Fig. 2a,b). Results for malignant tumours are summarised in Table 1.

\section{Group 2}

Seventy-one cases confirmed by biopsy were classified as malignant (BI-RADS IV-V), and 29 cases were categorised as most likely benign (BI-RADS III). Histological confirmation by vacuum biopsy was also performed at patients' expressed request and in cases of malignancy or borderline lesions also by surgery $(82 / 100)$.

\section{Histological appraisal}

\section{Benign lesions}

Histological finding detected four cases with a scar or intraductal inflammatory origin or ductal lesions, two cases of papillomas, five cases of fibrocystic mastopathy and 18 cases of fibroadenomas.
Fig. 2 a, b Effect on visibility of calcifications and delineation of tumour margin a Fundamental B mode. b Tissue harmonic imaging (THI)+cross beam low + speckle reduction imaging (SRI) level 1. Well-differentiated invasive ductal carcinoma with microcalcification and infiltration better seen in cross beam+SRI 2. There is less attenuation retrotumoral due to spatial compounding (SC)
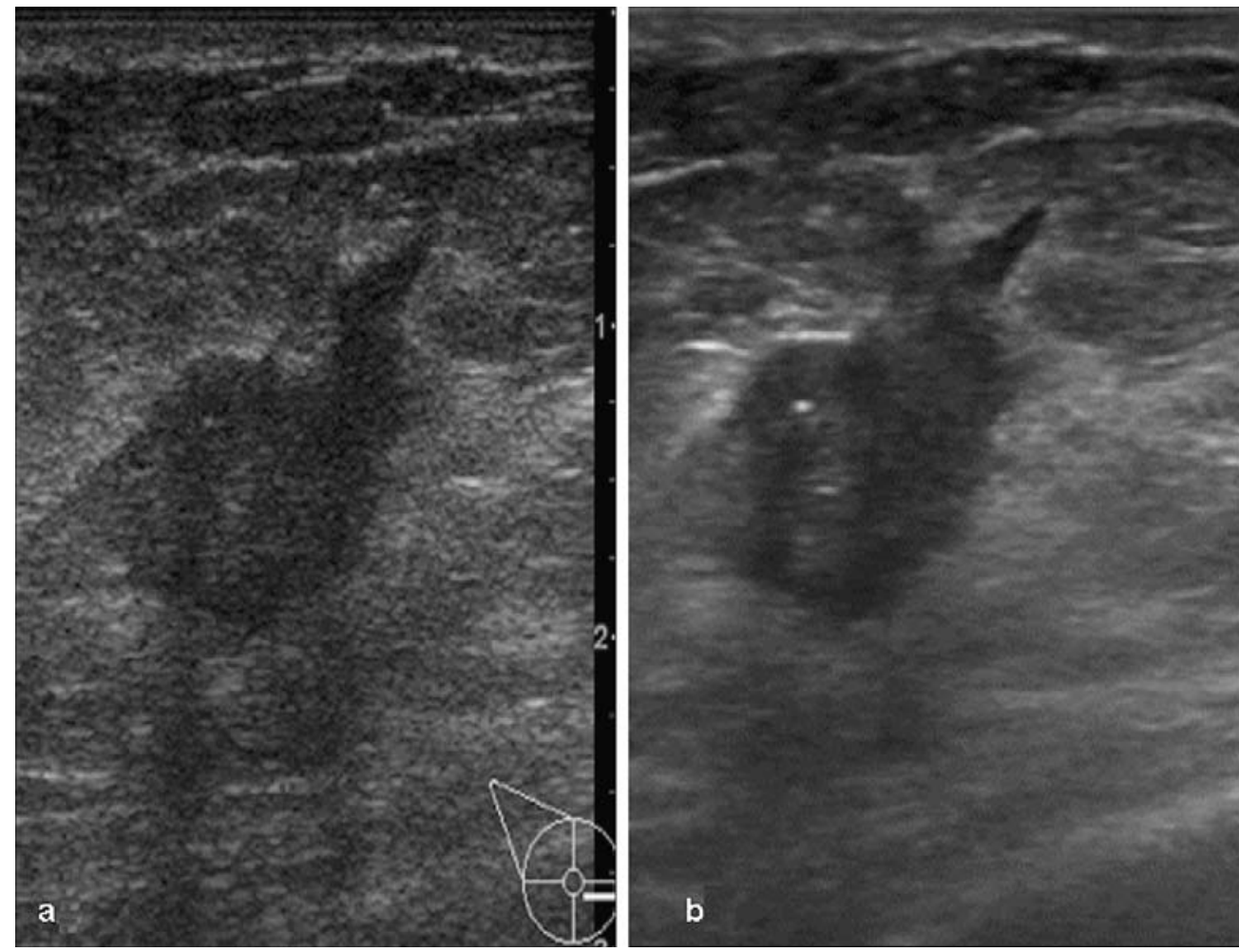
Table 1 Results of comparison of fundamental versus combination of tissue harmonic imaging (THI), spatial compounding (SC) (cross beam: level low) and speckle reduction imaging (SRI) level 1

\begin{tabular}{lrrr}
\hline Criteria & -1 & 0 & +1 \\
\hline Margin & 1 & 11 & 12 \\
Infiltration (existent in 19/24 malignant cases) & 1 & 3 & 15 \\
Echo pattern/tissue components & 3 & 10 & 11 \\
Calcifications & 1 & 2 & 3 \\
Posterior lesion boundary & 1 & 7 & 16 \\
Posterior acoustic features & 9 & 9 & 6
\end{tabular}

Score : $-1=$ fundamental superior, $0=$ no difference, $+1=$ combination superior

\section{Malignant lesions}

Histological findings revealed 11 ductal carcinomas in situ (DCIS), two lobular carcinomas in situ (LCIS) and 58 cases of ductal invasive carcinomas. Sonomorphology defined in particular the extent of the tumour, marginal contours, change in tissue architecture, possible infiltration of surrounding tissue, intraductal spreading, altered echogenicity of lesions with possible dorsal sound enhancement or attenuation and unchanged compressibility. In PD, CM enhancement with harmonic imaging additionally enabled detection of peritumoral or intratumoral and irregular vessels penetrating the tumour as well as increased tumour vascularisation in intermittent dynamic appraisal. In particular, irregular penetrating vessels and prolonged en- hancement for more than $5 \mathrm{~min}$ were found in only malignancies (65/71 cases: Fig. 3a-d, Fig. $4 a-d$ and Fig. 5a-f). In six cases of DCIS with a maximum diameter of $5 \mathrm{~mm}$ and low grading (G I), increased vascularisation could not be demonstrated, even after CM enhancement. All were detected as small lesions using a combination of THI with SC. Five out of six showed tiny peritumoral vessels after contrast administration. Proliferating fibroadenomas and scars may show an increased peritumoral vascularisation but did not show prolonged enhancement in harmonic imaging with PD (Table 2).

The results of our own investigations indicate that the tumours can be identified better during biopsy in echoinhomogeneous tissue using $\mathrm{CHI}$ in biopsies of smaller tumours under $10 \mathrm{~mm}$ in size. This enables false negative findings to be avoided in the histopathological investigations. After injection of CM, satellite foci and lymph nodes may also show increased vascularisation, which is manifested especially in harmonic imaging with PD.

\section{Discussion}

After clinical examination, breast US is the preferred method in symptomatic patients. In cases without symptoms, breast US is ascribed a higher sensitivity for detecting breast cancer in women with dense breast tissue, women under the age of 50 and high-risk women. Mammographically occult cancers can be detected by US in $10-40 \%$ of cases, depending on patients' breast density and age. For
Fig. 3 a-d Well-differentiated invasive ductal carcinoma (75-year-old woman, non-palpable mass) a Irregular tumour of $12 \mathrm{~mm}$ with hidden distal border in fundamental B scan and tissue harmonic imaging (THI). b, c In combination with cross beam and speckle reduction imaging (SRI), better delineation of the pectoral (distal) aspect, in particular, showing infiltration of the pectoral fascia. d Good visibility of the biopsy needle in THI and THI with crossbeam in three-dimensional (3D) technique
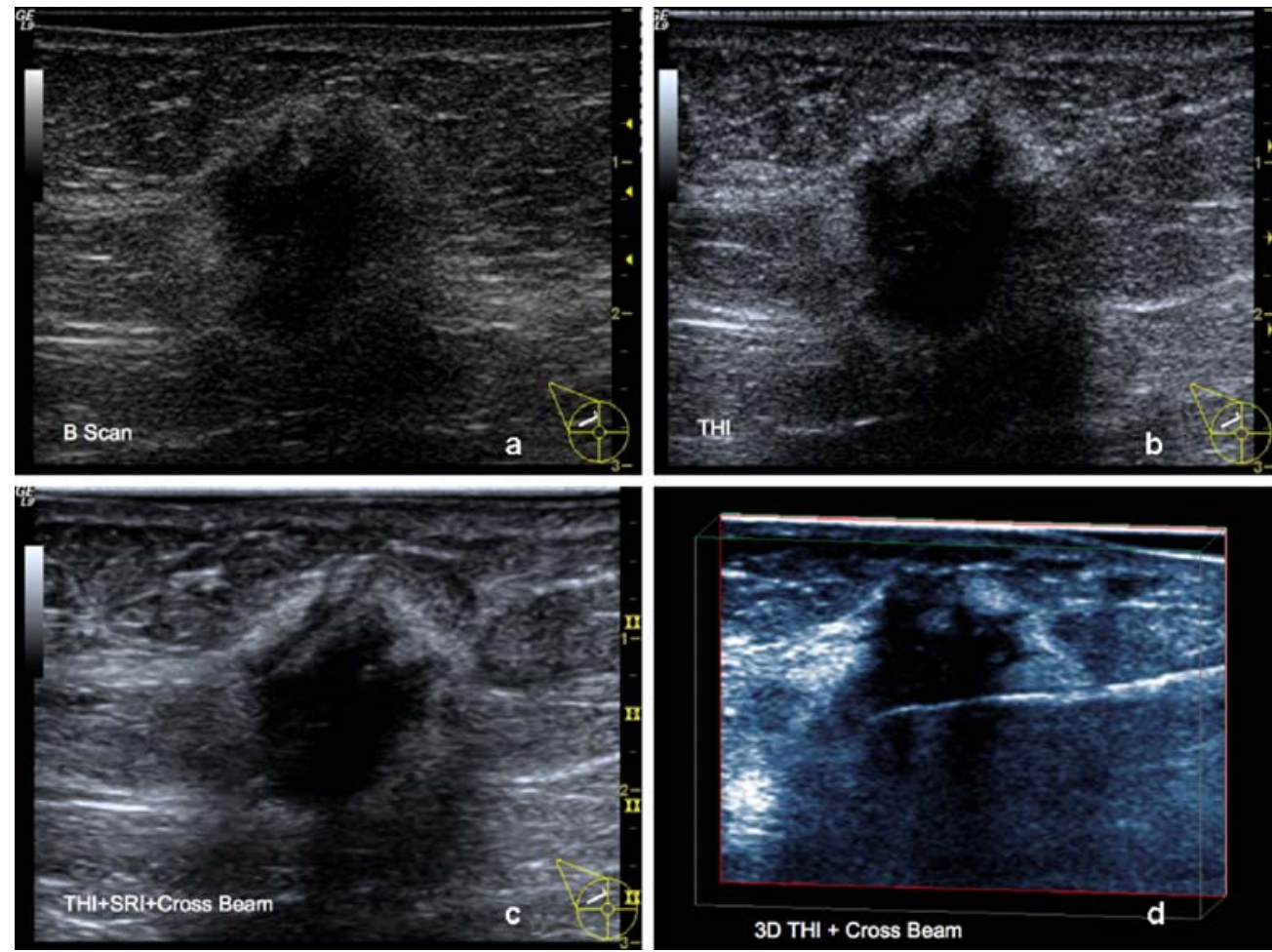
Fig. 4 a-d Same patient as Fig. 3 with invasive ductal carcinoma. a Unenhanced power Doppler (PD) shows few peripheral vessels. b-c After administration of $0.5 \mathrm{ml}$ Option, increasing enhancement within the lesion with prolonged persistence of enhancement up to 6 min is seen. d Beginning washout after $8 \mathrm{~min}$
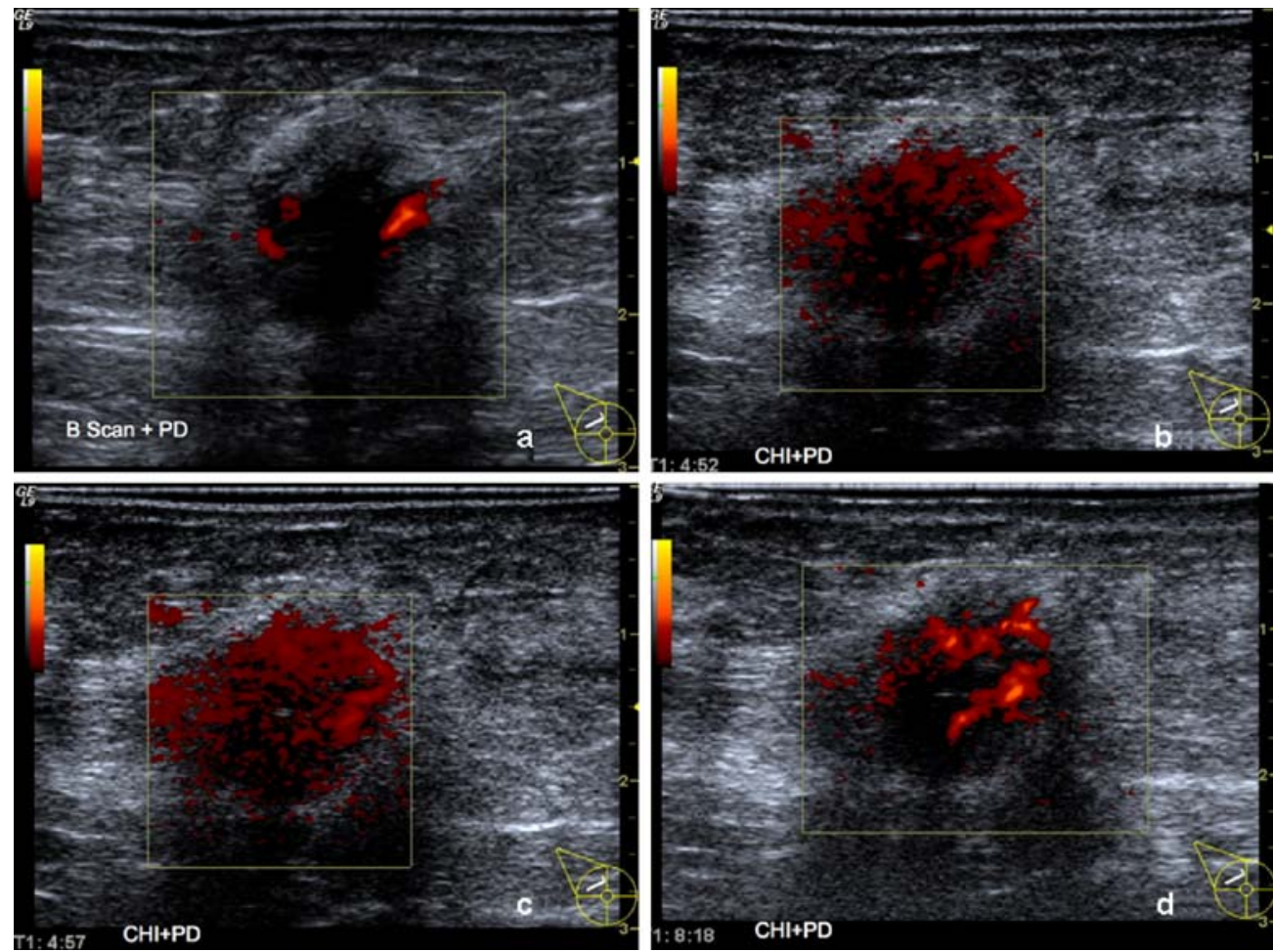

women without symptoms, breast US should be mandatory and complementary to mammography in the case of breast density grade II [American College of Radiology (ACR)] or more [34]. US is now the more accurate imaging test in women 45 years or younger who present with breast symptoms and should be the initial imaging investigation $[5,7,35]$. In preoperative assessment of the local extent of breast cancer, US showed higher sensitivity for invasive cancer than for DCIS $[8,35,41]$. In nonfatty breasts, US and MRI were more sensitive than mammography for detecting invasive cancer, but both MRI and US involved the risk of overestimating tumour extent $[4,7-9,12,35]$. Mammographic sensitivity decreased from $100 \%$ in fatty breasts to $45 \%$ in extremely dense breasts.

The respective diagnostic accuracy of the different breast imaging modalities, i.e. mammography $(\mathrm{Mx})$, high-resolution breast US, and dynamic contrast-enhanced breast scanning (MRI) regarding early diagnosis of familial (hereditary) breast cancer were investigated. Breast cancer detection rates were: Mx: $42 \%$, US $25 \%$ and MRI $83 \%$, and positive predictive values were: Mx $29 \%$, US $30 \%$ and MRI 43\% [8].

THI in combination with digital encoding of the US beam is an increasingly common option for B-mode imaging. It reduces reverberations, sidelobe effects and speckle. Both image contrast and lateral resolution are improved in harmonic mode compared with conventional fundamental US [36]. First applications are reported for liver disease [37], lymph node enlargement in children [38] and patients with pancreatitis. Benefits of harmonic imaging are more apparent in obese patients, improving accuracy of diagnosis from $60 \%$ to $80 \%$ [39]. To determine the impact of THI on visualisation of focal breast lesions, Szopinski et al. [21] performed a prospective study on 219 female patients. THI improved the grey scale contrast between fatty tissue and breast lesions in 230 lesions $(90.6 \% ; p<0.001)$ compared with fundamental frequency images. Contrast improvement was greater in breasts with predominantly fatty or mixed (fatty/glandular) composition than in predominantly glandular breasts. Overall conspicuousness, lesion border definition, lesion content definition and acoustic shadow conspicuousness were improved or equal in the harmonic mode for all lesions.

After optimising the system settings, our results showed better delineation of tumour borders and infiltration with the combination of SC and SRI. Better differentiation of tissue structure was seen in the combination of THI and SRI; microcalcifications could be depicted best with a combination of harmonic mode, SRI and SC [19, 27, 40]. Retrotumoral tissue and the posterior lesion boundary of tumours are significantly better identified with high values of SC. One must be aware of the potential problem that the typical posterior acoustic enhancement is significantly reduced, with the result that a cyst can be misinterpreted as a solid tumour.

THI and CHI can be helpful for detecting tumours less than $10 \mathrm{~mm}$ in size in echo-inhomogeneous dense tissue, for characterising tumour morphology with dynamic enhancement studies and for performing a definitive core needle biopsy or vacuum-assisted biopsy in cases of 
Fig. 5 a, b Ductal carcinoma in situ (DCIS) (35-year-old woman, no palpable mass). Cervical carcinoma, breast cancer in mother and sister. No clinical signs. Tumour proven by vacuum-assisted biopsy (DCIS) a Fundamental (B scan). b Tissue harmonic imaging (THI) showing a small, distinct, irregular mass of $8 \mathrm{~mm}$.

c-f Same patient as a, b. c Power Doppler (PD) before administration of contrast. d Pronounced visibility of the feeding vessel in the early phase. e Late and persistent enhancement of the tumour typical for malignant breast tumours. $\mathbf{f}$ Better delineation of the tumour even after $14 \mathrm{~min}$ in contrast harmonic imaging (CHI) mode
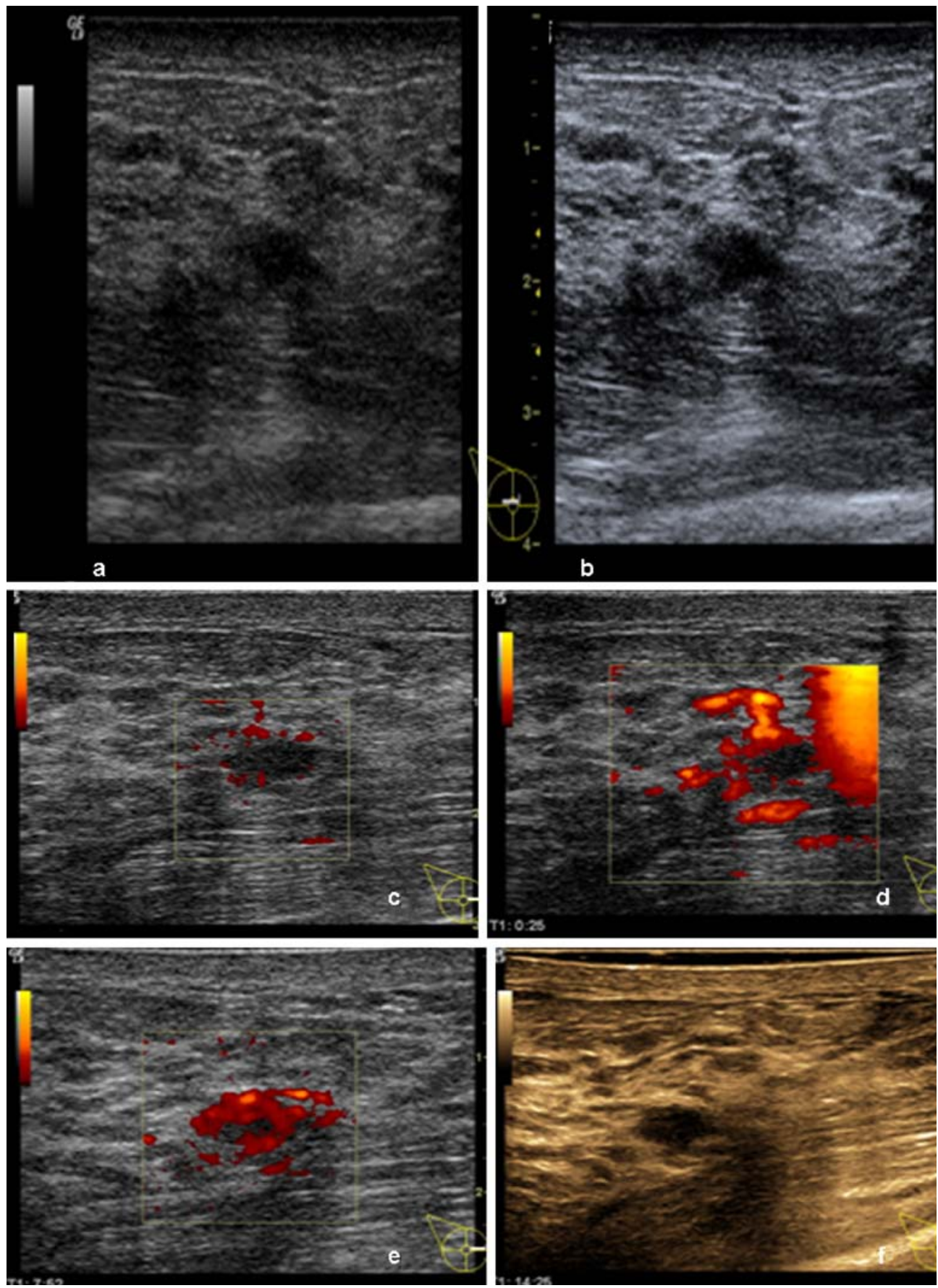

tumour lesions less detectable in fundamental B scan or tumours located in the deep tissue near the intercostal muscles. Harmonic imaging in combination with SRI and cross beam can facilitate detection of intraductal tumours such as papillomas. With 3D imaging, the needle could be more easily placed in the middle of these small intraductal tumours during biopsies [15].

Various criteria of image morphology have proved useful in appraising whether a breast tumour is benign or malignant on the basis of US. Smooth marginal contour, dorsal US amplification, displacement margin, bilateral US extinction and absence of structural discontinuity in that order rather indicate a benign lesion. An echodense border, tumour outliers, jagged marginal contour, dorsal extinction and structural discontinuity point to malignant lesions. In consequence of the increasing improvements in tumour detection and morphological imaging, sensitivity $<80 \%$ and specificities $<95 \%$ can be achieved with US in tumour diagnostics $[5,7,20,21,35]$. An even higher diagnostic certainty is aspired to. This can already be attained with the 
Table 2 Comparison of benign tumours versus carcinomas. Vascularisation after administration of CM [0.5 ml Optison]

\begin{tabular}{|c|c|c|c|c|}
\hline & \multicolumn{2}{|c|}{ Benign tumours $(n=29)$} & \multicolumn{2}{|c|}{ Carcinomas $(n=71)$} \\
\hline & Power Doppler & Power Doppler $+\mathrm{CM}$ & Power Doppler & Power Doppler $+\mathrm{CM}$ \\
\hline \multicolumn{5}{|l|}{ Early phase (1-5 min) } \\
\hline Marginal vessels & $11 / 29(38 \%)$ & $24 / 29(83 \%)$ & $43 / 71(60 \%)$ & $67 / 71(94 \%)$ \\
\hline Penetrating vessels & $4 / 29(14 \%)$ & $12 / 29(41 \%)$ & $28 / 71(39 \%)$ & $65 / 71(91 \%)(p<0.05)$ \\
\hline Central vessels & $0 / 29(0 \%)$ & $6 / 29(20 \%)$ & $21 / 71(29 \%)$ & $48 / 71(68 \%)(p<0.05)$ \\
\hline \multicolumn{5}{|l|}{ Late phase $(5-20 \mathrm{~min})$} \\
\hline Diffuse enhancement & $0 / 29$ & $0 / 29$ & $0 / 71$ & $65 / 71(91 \%)(p<0.01)$ \\
\hline
\end{tabular}

Marginal vessels are seen in both groups in a high percentage. Most carcinomas show diffuse enhancement through the late phase (statistical evaluation with the Fisher's exact test, significant differences $p<0.05$ )

combination of mammography and breast US, especially in dense and inhomogeneous glandular tissue [2, 7, 22, 35].

Tumour lesions of BI-RADS IV and V have to be clarified by biopsy, in particular, punch biopsy or vacuum biopsy. Consequently, US-guided biopsy is appropriate when it allows unequivocal lesion identification. USguided interventions are required for further clarification in mammography occult findings and preoperative wire marking. In our own investigations, harmonic imaging was employed for definitive preoperative wire marking, to carry out a US-guided vacuum biopsy and also with CM enhancement for improved tumour detection.

Additional Doppler methods have been used to analyse breast tumour vascularity $[42,43]$. Detection of penetrating or central vessels proved to be an accurate sign of malignancy $[12,13,17,22,24,25,29,31]$. With the application of CM (Levovist), additional vessels were detected within the lesions, increasing diagnostic accuracy. Sensitivity of colour-coded US was improved from $64 \%$ up to $86 \%$ using the echo signal amplifier. Specificity was $86 \%$ without and $82 \%$ with echo signal amplifier. MRI was found to have a sensitivity of $100 \%$ and a specificity of up to $82 \%$ [12].

Our study with a modified technique of CM-enhanced PD and THI showed better visualisation of tumour vascularisation after bolus application of Optison. Persistent enhancement after $5 \mathrm{~min}$ was characteristic for malignant tumours, enabling tumour characterisation as has hitherto only been known from CM-enhanced MRI $[4,12,16,18,24,29-31,44]$.
When using $\mathrm{CM}$ containing protein, special attention must be paid to an allergic predisposition and kidney function disorder. The use of second-generation $\mathrm{CM}$ necessitates a change in $\mathrm{MI}$ and imaging with harmonic imaging. However, the modified technique allows a differentiated diagnosis of tumour morphology and vascularisation $[4,12,25,29,44,45]$.

To improve the detection and assessment of intratumoral vessels in malignant breast tumours, techniques to enhance visualisation of vessels within focal lesions have been developed and automated. These techniques focus on the early perfusion phase following CM administration. Contrast-enhanced MRI allowed semiquantitative assessment of the kinetics of contrast media [4, 18, 31]. Artefacts caused by pulsation and patient movement should be avoided. More frequently, 3D postprocessing has become important in assessment of vasculature. CHI US is already used to distinguish scars from recurrent tumours as well as in the optimisation of US-guided biopsies [14, 15, 20, 32].

\section{Conclusion}

In conclusion, we feel confident that US using THI and $\mathrm{CHI}$ technologies is a valuable tool for evaluating focal breast lesions, in particular in mammography-dense breasts. If utilised extensively, it will be a cost-effective tool to facilitate detection and differentiation of malignant breast lesions in early, well-treatable stages and will thus help in reducing breast cancer mortality.

\section{References}

1. Baker JA, Kornguth PJ, Soo MS, Walsh R, Mengoni P (1999) Ultrasound of solid breast lesions: observer variability of lesion description and assessment. AJR Am J Roentgenol 172:1621-1625
2. Bazzocchi M, Facecchia I, Zulani C, Puglisi F, Di Loreto C, Smania S (2000) Diagnostic Imaging of lobular carcinoma of the breast: mammographic, ultrasonographic and MR findings. Radiol Med (Torino) 100:436-443
3. Berg WA, Campassi CI, Ioffe OB (2003) Cystic lesions of the breast: sonographic-pathologic correlation. Radiology 227:183-191

4. Delorme S (1998) Evaluating tumor vascularization with Doppler ultrasound. Radiologe 38:335-343 
5. Houssami N, Irwig L, Simpson J, McKessar M, Blome S, Noakes J (2003) Sydney breast imaging accuracy study: comparative sensitivity and specificity of mammography and ultrasound in young women with symptoms. AJR Am J Roentgenol

181:1423-1425

6. Hunt KA, Sickles EA (2000) Effect of obesity on screening mammography: outcomes analysis of 88346 examinations. AJR Am J Roentgenol 174 (5):1251-1255

7. Kolb TM, Lichy J, Newhouse JH (2002) Comparison of the performance of screening mammography, physical examination, and breast US and evaluation of factors that influence them: an analysis of 27825 patients evaluations. Radiology 225:165-175

8. Kuhl C, Schrading S, Weigel S, NussleKugele K, Sittek H, Arand B, Morakkabati N, Leutner C, Tombach B, Nordhoff D, Perlet C, Rieber A, Heindl W, Brambs H, Schild H (2005) The "EVA" Trial: evaluation of the efficacy of diagnostic methods (Mammography, Ultrasound, MRI) in the secondary and tertiary prevention of familial breast cancer. Preliminary results after the first half of the study period. Röfo 177:818-827

9. Metha TS (2003) Current uses of ultrasound in the evaluation of the breast. Radiol Clin North Am 41: 841-856

10. Pavic D, Koomen M, Kuzmiak C, Pisano ED (2003) Ultrasound in the management of breast disease. Curr Womens Health Rep 3:156-164

11. Svensson WE (2000) The value of ultrasound scanning in breast disease. Hosp Med 61:233-239

12. Aichinger U, Schultz-Wendtland R, Kramer S, Lell M, Bautz W (2002) Scar or recurrence-comparison of MRI and color-coded ultrasound with echo signal amplifiers. Röfo 174:1395-1401

13. Alamo L, Fischer U (2001) Contrastenhanced color Doppler ultrasound characteristics in hypervascular breast tumors: comparison with MRI. Eur Radiol 11:970-977

14. Cheung YC, Wan YL, Chen SC, Lui $\mathrm{KW}, \mathrm{Ng} \mathrm{SH}$, Yeow KW, Lee KF, Hsueh S (2002) Sonographic evaluation of mammographically detected microcalcifications without a mass prior to stereotactic core needle biopsy. J Clin Ultrasound 30:323-331

15. Lell M, Wenkel E, Aichinger U, Schultz-Wendtland R, Bautz W (2004) 3D ultrasound in core breast biopsy. Ultraschall Med 25:126-130
16. Reinikainen H, Rissanen T, Paivansalo M, Paakko E, Jauhianinen J, Suramo I (2001) B-Mode, power Doppler and contrast-enhanced power Doppler ultrasound in the diagnosis of breast tumors. Acta Radiol 42:106-113

17. Schroeder RJ, Bostanjoglo M, Hidajat N, Rademaker J, Rottgen R, Maurer J, Felix R (2002) Analysis of vascularity in breast tumors-comparison of high frequency ultrasound and contrast-enhanced color harmonic imaging. Rofo 174:1132-1111

18. Schroeder RJ, Bostanjoglo M, Rademaker J, Maeurer J, Felix R (2003) Role of power Doppler techniques and ultrasound contrast enhancement in the differential diagnosis of focal breast lesions. Eur Radiol 13:68-79

19. Seo BK, Oh YW, Kim HR, Kim HW, Kang CH, Lee NJ, Kim JH, Park BJ, Cho KR, Lee JY, Lee KY, Bae JW (2002) Sonographic evaluation of breast nodules: comparison of conventional, real-time compound, and pulseinversion harmonic images. Korean J Radiol 3:38-44

20. Soo MS, Baker J, Rosen EL (2003) Sonographic detection and sonographically guided biopsy of breast microcalcifications. AJR Am J Roentgenol 180:941-948

21. Szopinski KT, Pajk AM, Wysocki M, Amy D, Szopinska M, Jakubowski W (2003) Tissue harmonic imaging: utility in breast ultrasound. J Ultrasound Med 22:479-487; quiz 488-479

22. Yang WT, Metreweli C, Lam PKW, Chang J (2001) Benign and malignant breast masses color power Doppler US and axillary nodes: evaluation with echo-enhanced color power Doppler US. Radiology 220:795-802

23. Jung EM, Clevert DA, Rupp N (2003) Contrast-enhanced ultrasound with Optison in percutaneous thermoablation of liver tumors. Rofo 175: 1403-1412

24. Jung EM, Jungius KP, Lenhart M, Dengler M, Becker H, Rupp N (2004) Tissue harmonic imaging (THI) and contrast harmonic imaging $(\mathrm{CHI})$ for sonographically guided vacuum-assisted biopsy of mammographically occult breast tumors. Rofo 176: 1416-1422

25. Lamuraglia M, Lassau N, Garbay J, Mathieu M, Rouzier R, Jaziri S, Roche A, Leclere J (2005) Doppler US with perfusion software and contrast medium injection in the early evalution of radiogreyuency in breast canccer recurrences: a prospective phase II study. Eur J Radiol 6: DOI 10.1016/j. ejrad.2005.06.003
26. Kubota K, Ogawa Y, Nishigawa T, Yoshida S (2003) Tissue harmonic imaging ultrasound of the axillary lymph nodes: evaluation of response to neoadjuvant chemotherapy in breast cancer patients. Oncol Rep 10: 1911-1914

27. Rosen EL, Soo MS (2001) Tissue harmonic imaging ultrasound of breast lesions: improved margin analysis, conspicuity, and image quality compared to conventional ultrasound. Clin Imaging 25:379-384

28. Denis F, Bougnoux P, de Poncheville L, Prat M, Catroux R, Tranquart F (2002) In vivo quantification of tumor vascularization assessed by Doppler ultrasound in rat mamma tumors. Ultrasound Med Biol 28:431-437

29. Huber S, Vesely M, Zuna I, Delorme S, Czembierek H (2001) Fibroadenomas: computer-assisted quantitative evaluation of contrast-enhanced power Doppler features and correlation with histopathology. Ultrasound Med Biol 27:3-11

30. Menna S, Di Virgilio MR, Burke P (1999) Ultraultrasound contrast media Levovist and power Doppler in the study of the breast. Methodology, vascular morphology and automatic enhancement quantification with wash-in and wash-out curves. Radiol Med (Torino) 97:472-478

31. Pudszuhn A, Marx CH, Malich A, Fleck M, Schneider A, Kaiser WA (2003) Prospective analysis of quantification of contrast media enhanced power Doppler ultrasound of equivocal breast lesions] Computer-assistierte Quantifizierungsanalyse der kontrastmittelverstärkten Power DopplerSonographie bei unklaren Herdbefunden der Mamma. Rofo 175:495-501

32. Jung EM, Clevert DA, Lutz R, Kett H, Rupp N (2002) Preoperative wire localisation of breast lesions by tissue harmonic imaging (THI) ultrasound. Rofo 174:1121-1125

33. Stavros AT (2004) Breast ultrasound. Lippincott Williams and Wilkens, Philadelphia

34. Hille H, Vetter M, Hackeleor BJ (2004) Re-evaluating the role of breast ultrasound in current diagnotics of maligne breast lesions. Ultraschall Med 25 (6):411-417

35. Berg WA, Gutierez L, NessAiver MS, Carter WB, Bhargavan M, Lewis RS, Ioffe OB (2004) Diagnostic accuracy of mammography, clinical examination, US, and MR imaging in preoperativ assesment of breast cancer. Radiology 233:830-849 
36. Desser TS, Jeffrey RB (2001) Tissue harmonic imaging techniques: physical principles and clinical applications. Semin Ultrasound CT MR 22:1-10

37. Hann LE, Bach AM, Cramer LD, Siegel D, Yoo HH, Garcia R (1999) Hepatic ultrasound: comparison of tissue harmonic and standard ultrasound techniques. AJR Am J Roentgenol 173:201-206

38. Dybiec E, Brodzisz A, Pietka M, Obloza M, Niedzielska G, Kowalczyk J, Wieczorek P (2002) The application of ultrasound contrast, 3D imaging and tissue harmonic imaging in the differential diagnosis of lymph nodes enlargement in children. Ann Univ Mariae Curie Sklodowska [Med] 57:131-142
39. Garg M, Sandhu M, Sood B, Lal A, Suri S, Bhasin D (2004) Tissue harmonic imaging in the evaluation of acute pancreatitis. Australas Radiol 48:117-122

40. Liu DL, Waag RC (1997) Harmonic amplitude distribution in a wideband ultrasonic wavefront after propagation through human abdominal wall and breast specimens. J Acoust Soc Am 101:1172-1183

41. Yang WT, Tse GM (2004) Sonographic, mammographic, and histopathologic correlation of symptomatic ductal carcinoma in situ. AJR Am J Roentgenol 182:1001-1010

42. Stuhrmann M, Aronius R, Schietzel M (2000) Tumor vascularity of breast lesions: potential and limits of contastenhanced Doppler ultrasound. AJR Am J Roentgenol 175:1585-1589
43. Madjar H, Jellins S, Schillinger H, Hillemanns HG (1986) Differenzierung von Mammacarcinomen durch cwDoppler ultraschall. Ultraschall Med 7:183-184

44. Madjar H, Sauerbrei W, Pröpeler HJ, Wolfahrt R, Gufler H (1997) Color doppler and duplex flow analysis for classification of breast lesions. Gynaecol Oncol 64:392-403

45. Quaia E, D'Onofrio M, Palumbo A, Rossi S, Bruni S, Cova M (2006) Comparison of contrast-enhanced ultrasonography versus baseline ultrasound and contrast-enhanced computed tomography in metastatic disease of the liver: diagnostic performance and confidence. Eur Radiol DOI 10.1007/ s00330-006-0192-7 